\title{
Review of Two Approaches for the Care of Elderly Hip Fracture Patient
}

\section{Christopher A. Brown*1, Sami Mardam-Bey², John Boling², Steven Olson'1, Thomas Owens ${ }^{3}$ and Robert Zura' ${ }^{1}$}

${ }^{1}$ Duke University Medical Center, Department of Orthopedics, Durham North Carolina, USA

${ }^{2}$ Duke University Medical School, Durham North Carolina, USA

${ }^{3}$ Duke University Medical Center, Department of Medicine, Durham North Carolina, USA

\begin{abstract}
Introduction: Protocols for caring for elderly hip fracture patients vary among hospitals. The purpose of this study was to examine two different approaches of care for this patient population at our institution.

Methods: Retrospective review of 389 consecutive patients 65 years and older with the diagnosis of a lowenergy, hip fracture between January 2006 and May 2010. 227 consecutive patients for which a nonstandard method of admission (OP) to either medicine (133 patients) or orthopedic service (94 patients) were compared to a new plan of care (NP) that included 162 consecutive patients in which all patients were admitted to medicine. We evaluated perioperative complications rates, time to surgery (TTS), and length of hospital stay (LOS).

Results: There were no significant differences in LOS, TTS, 30 day re-admission, rapid response codes, perioperative complications or death between either model of care. The NP did demonstrate a significant increase in patients being transfused $(51.85 \%$ vs. $36.56 \%)$ and number of patients being diagnosed with Vitamin D deficiency $(42.12 \%$ vs. $20.70 \%)$.

Conclusion: While our change in protocol did not alter the rate of perioperative complications, nor did it provide quicker care or shorter hospital stays, it did provide more comprehensive osteoporosis care to our patients.
\end{abstract}

Keywords: Elderly hip fracture; Perioperative complications; Osteoporosis treatment.

\section{Introduction}

The incidence of hip fractures increases exponentially after the age of 65 and projections indicate that the number will rise over the coming decades because of an aging population [1]. By 2050 future estimates of the number of hip fracture may range from 458,000 to $1,037,000$ with the largest number occurring in females over the age of 65 [2]. The current annual cost for treatment is more than $\$ 9.8$ billion, and these costs are increasing at a time when control of health care costs is vital [3]. Hip fractures account for more inpatient hospital days than any other musculoskeletal injury, and represents 44 percent of all inpatient hospital days due to fractures [3]. Given the current health care environment these costs are significant.

In an attempt to provide better patient care for elderly patients with multiple co-morbidities who experience hip fractures some centers have created various forms of shared-responsibility services, multidisciplinary or hospitalist models of care for these patients. Reports indicate improvements in time to surgery, level of hospital care, length of stay, medical complications, co-morbid conditions, mortality, re-hospitalization rates, long-term care and hospital costs [4-12]. Despite these encouraging results, improved medical management, implementation of preventive strategies, exists growing concern about the inadequacy of osteoporosis care for these patients [13]. It is also still unclear what is the most efficient, cost-effective, and safe protocol for caring for these patients as care models can vary from hospital to hospital.

At our institution, patients with hip fractures historically have been admitted to either a medicine or orthopedics service with the other team in consultation. There was no systematic admission protocol and admission to medicine or orthopedics was at the discretion of the emergency department and each service's on call resident. It was our perception that this process was arbitrary and a potential cause of delay in the treatment. Additionally, due to an unexpected mortality of a patient on the orthopedic service, an admission protocol was standardized in an attempt to improve patient safety. In 2008, the Departments of Medicine and Orthopedics instituted a new mandate directing all patients over 65 years of age with femoral fractures be admitted directly to medicine service with orthopedic service providing consultation for surgical services.

This review was therefore undertaken to examine this new mandate, analyze its effectiveness, and determine if care had been improved.

\section{Patient and methods}

This study was performed at Duke University Medical Center in Durham, North Carolina. This study consisted of 389 consecutive patients 65 and older who were admitted to our institution with the diagnosis of hip fracture identified by current procedure terminology (CPT) codes $(27235,27236,27244,27245,27125)$ and International Classification of Disease Code (ICD-9) code of 820 between January 2006, and May 2010. The earlier approach (OP) was defined as all patients before October 2008 in which a nonstandard plan of care was used and included 227 patients. This OP included admission to either a medical service (133 patients) or an orthopedic service (94 patients). The new approach (NP) was defined as all patients admitted after October 2008 in which a new plan of care was implemented and included 162 patients. This NP involved direct admission of all hip

*Corresponding author: Christopher A. Brown, Department of Orthopedics, Duke University Medical Center, Durham North Carolina, Box 3269200 Trent Drive, Durham, NC 27710, USA, Tel: 919-668-4732; Fax: 919-681-7795; E-mail: christopher.a.brown@duke.edu

Received March 12, 2012; Accepted March 30, 2012; Published April 02, 2012

Citation: Brown CA, Mardam-Bey S, Boling J, Olson S, Owens T, et al. (2012) Review of Two Approaches for the Care of Elderly Hip Fracture Patient. J Trauma Treat 1:125. doi:10.4172/2167-1222.1000125

Copyright: ( 2012 Brown CA, et al. This is an open-access article distributed under the terms of the Creative Commons Attribution License, which permits unrestricted use, distribution, and reproduction in any medium, provided the original author and source are credited. 
fractures to a medicine service with preoperative medical evaluation, medical optimization, and postoperative medical management with consultation by orthopedic service. Patients were excluded if they had sustained multiple or high-energy injuries and/or non-orthopedics trauma-related injuries. Figure 1 illustrates the number of patients in each care model.

\section{Data collection}

A retrospective chart review was performed with use of the hospital electronic medical record system to obtain data regarding patient characteristics that included age, sex, race, body mass index(BMI), ASA score (American Society of Anesthesiologists), co-morbidity, type of fracture, type of fracture treatment, pre-fracture mobility status, date and time of admission, date of discharge, admitting service, readmission to hospital within 30 days for reason, rapid response code (RRT), transfusion, pre-fracture and post-fracture supplementation with Vitamin D, and any postoperative complications or death. All patients were assigned an ASA class as either part of their anesthesia preoperative assessment or calculated based on the typical five tier system [14]. Type of medical co-morbidities that were recorded included cerebral vascular disease, dementia, pre-operative urinary tract (UTI), renal insufficiency, diabetes mellitus, hypertension (HTN), congestive heart failure (CHF), coronary artery disease (CAD), chronic obstructive pulmonary disease( COPD). Pre-fracture functional mobility status was defined as being either a independent, cane assisted, walker assisted, or wheelchair ambulator. Fracture type was recorded as either intracapsular which included femoral neck or extracapsular which included basicervical and peritrochanteric fractures. Type of fracture treatment included hemiarthroplasty, intramedullary nail, dynamic hip screw, percutaneous pinning, total hip arthroplasty, girdlestone, or non-operative treatment. Perioperative complications were recorded if a patient had presence of pressure sores, pulmonary embolus (PE), deep vein thrombosis (DVT), COPD exacerbation, new UTI, pneumonia, CHF exacerbation, myocardial infarct (MI), wound complications, renal insufficiency, delirium, or death. Admission service was either medicine or orthopedic service. Time of surgery was defined as start of anesthesia.

From this information LOS (length of hospital stay defined as time from admission to discharge) and TTS (time to surgery defined as time from admission to start of anesthesia) was calculated.

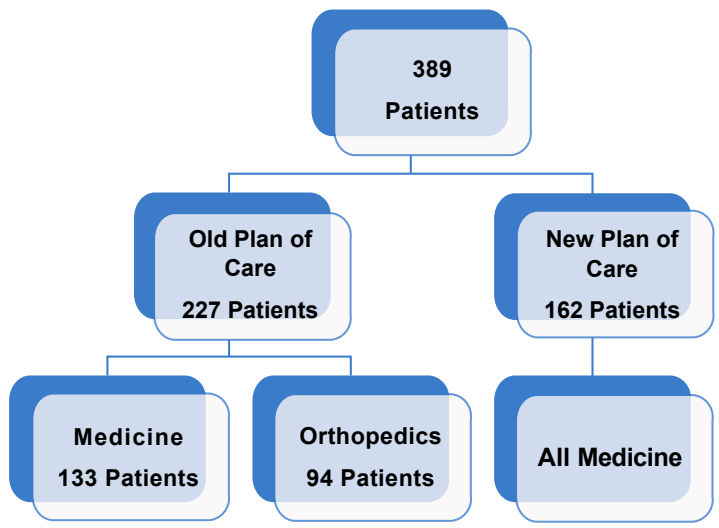

Figure 1: This diagram illustrates the breakdown of number of patients examined in this study and their respective plan of care and admission service.

\section{Statistical analysis}

Patient characteristics were summarized with the frequency and percentage of patients for each care plan. A Chi Square analysis was used to compare these patient characteristics to verify that each plan of care sample population was similar in comparison. Subgroup analysis for patients admitted under OP was done to examine each respective group's initial patient characteristics and perioperative complications. A Chi Square analysis was used to compare the frequency of perioperative complications, re-admission, RRT, and Vitamin D supplementation between NP and OP. The student $t$ test for two independent groups was used to examine the role that each plan of care or subgroup has on mean TTS and LOS. Analysis of variance was used to examine mean differences when three or more means were compared. Significant effects were analyzed using the Duncan multiple range post-hoc test.

\section{Results}

Preoperative patient characteristics are demonstrated in Table 1 for the two protocols. We found that there were no significant differences $(p<0.05)$ between the OP and NP with regard to frequency of age group, race, sex, ASA level, fracture type, pre-fracture functional status, and preoperative co-morbidities (with the exception of PVD). Mean age and BMI were also similar. Hemiarthoplasty (37.62\%), intramedullary nail (31.62\%), dynamic hip screw (20.57\%), and percutaneous pinnng (9.51\%) were the most common surgical method of treatment used for these patients. We did not find a difference between TTS between intracapsular and extracapsular fractures, 26.84 hours (std dev 27.91) vs. $24.94($ std $\operatorname{dev} 29.45)(\mathrm{p}=0.53)$ respectively.

To better understand which type of patients were being admitted to medicine and orthopedics under the OP, a subgroup analysis was undertaken. Table 2 illustrates the subgroup analysis and indicates that those initially admitted to the medicine service had increased frequency of co-morbidities and ASA 4 patients when compared to those admitted to the orthopedic service. However, those patients admitted to the orthopedic service were not without co-morbidities with $11.7 \%$ having CHF, 27.6\% CAD, 17.9\% CVD, 68.1\% HTN, 10.6\% COPD, $17 \%$ UTI, $12.8 \%$ Diabetes. $79.8 \%$ were classified as ASA 3 and $7.5 \%$ as ASA 4 .

We found that there were no significant differences in perioperative complications between the NP and the OP in regard to 30 day readmission, RRT, postoperative delirium, MI, CHF exacerbation, pneumonia, COPD exacerbation, PE, DVT, decubitus ulcers, surgical wound complications, UTI or death (Table 3). The NP did demonstrate a significant increase frequency of patients being transfused $(51.85 \%$ vs. $36.56 \%)$ and postoperative renal insufficiency (23.46\% vs. $15.42 \%)$. Perioperative complications for the two subgroups admitted under the OP demonstrated that patients admitted to the medicine had an increased frequency of peri-operative complications in 4 categories (Table 4). There was a significant increase frequency of CHF exacerbation, pneumonia, and renal insufficiency, COPD exacerbation, but no significantly difference occurred with regard to mortality, RRT, re-admission, PE, DVT, decubitus ulcer, wound complications, transfusion, UTI, or Delerium ( $\mathrm{p}>0.05)$

Table 5a illustrates the NP vs. OP mean TSS and LOS. The mean TTS for the NP was 2.57 (27.02 Vs. 24.45) hours shorter, but using the student $\mathrm{t}$ test, this difference is not significant. There was no significant difference in mean LOS with the NP mean LOS 7.15 (std dev 4.26 days) and the OP mean LOS 6.83 (std dev 4.52 days). When comparing the 
Citation: Brown CA, Mardam-Bey S, Boling J, Olson S, Owens T, et al. (2012) Review of Two Approaches for the Care of Elderly Hip Fracture Patient. J Trauma Treat 1:125. doi:10.4172/2167-1222.1000125

Page 3 of 7

\begin{tabular}{|c|c|c|c|c|c|}
\hline Patient Characteristics & New \#(\% Approach) & Old \#(\% Approach) & Total \#(\%) & Chi Square & P value \\
\hline Number & 162 & 227 & 389 & & \\
\hline Age (Mean Years) & 82.31(std dev 7.87) & 82.27(std dev 7.27) & 82.29(std dev 7.52) & & \\
\hline BMI(mean) & 23.50(std dev 4.25) & 24.54(std dev 4.89) & 24.05(std dev 4.61) & & \\
\hline Age per Group & & & & 0.7382 & 0.6913 \\
\hline $65-75$ years & $33(20.37 \%)$ & $43(18.94 \%)$ & $76(19.54 \%)$ & & \\
\hline $76-85$ years & $65(40.12 \%)$ & $101(44.49 \%)$ & $166(42.67 \%)$ & & \\
\hline$>86$ years & $64(39.51 \%)$ & $83(36.56 \%)$ & $147(37.79 \%)$ & & \\
\hline Race & & & & 4.2753 & 0.2332 \\
\hline $\mathbf{w}$ & $135(83.3 \%)$ & $186(82.3 \%)$ & $321(82.7 \%)$ & & \\
\hline B & $26(16.05 \%)$ & $36(15.93 \%)$ & $62(15.98 \%)$ & & \\
\hline other & $1(0.65 \%)$ & $5(1.77 \%)$ & $6(1.32 \%)$ & & \\
\hline Sex & & & & 3.4044 & 0.065 \\
\hline M & $60(37.04 \%)$ & $64(28.19 \%)$ & $124(31.88 \%)$ & & \\
\hline $\mathbf{F}$ & $102(62.96 \%)$ & $163(71.81 \%)$ & $265(68.12 \%)$ & & \\
\hline ASA & & & & 0.0399 & 0.9803 \\
\hline 1 & 0 & 0 & 0 & & \\
\hline 2 & $12(7.4 \%)$ & $17(7.49 \%)$ & $29(7.455 \%)$ & & \\
\hline 3 & $118(73.84 \%)$ & $167(73.57 \%)$ & $285(73.33 \%)$ & & \\
\hline 4 & $32(19.75 \%)$ & $43(18.94 \%)$ & $75(19.28 \%)$ & & \\
\hline 5 & 0 & 0 & 0 & & \\
\hline \multicolumn{6}{|l|}{ Fracture type } \\
\hline Intracapsular & $76(46.91 \%)$ & $113(49.78 \%)$ & $189(48.59 \%)$ & 0.3109 & 0.5771 \\
\hline Extracapsular & $86(53.09 \%)$ & $114(50.22 \%)$ & $200(51.41 \%)$ & & \\
\hline \multicolumn{6}{|l|}{ Pre-fracture Functional Status } \\
\hline Independent & $69(43.40 \%)$ & $79(35.59 \%)$ & $148(38.85 \%)$ & 2.7372 & 0.4339 \\
\hline Cane & $27(16.98 \%)$ & $48(21.62 \%)$ & $75(19.69 \%)$ & & \\
\hline Walker & $55(34.59 \%)$ & $84(37.84 \%)$ & $139(36.48 \%)$ & & \\
\hline Wheelchair & $8(5.03 \%)$ & $11(4.95 \%)$ & $19(4.99 \%)$ & & \\
\hline \multicolumn{6}{|l|}{ Preoperative Co-morbidities } \\
\hline Dementia & $56(34.57 \%)$ & $65(28.63 \%)$ & $121(31.11 \%)$ & 1.5531 & 0.2127 \\
\hline Congestive Heart Failure & $31(19.14 \%)$ & $57(25.11 \%)$ & $88(22.62 \%)$ & 1.9276 & 0.165 \\
\hline Coronary Heart Disease & $59(36.42 \%)$ & $93(40.97 \%)$ & $152(39.07 \%)$ & 0.8219 & 0.3646 \\
\hline Peripheral Vascular Disease & $39(24.07 \%)$ & $27(11.89 \%)$ & $66(19.97 \%)$ & 9.9546 & 0.0016 \\
\hline Cerebral Vascular Disease & $24(14.81 \%)$ & $40(17.62 \%)$ & $64(16.45 \%)$ & 0.5416 & 0.4618 \\
\hline Hypertension & $127(78.4 \%)$ & $170(74.89 \%)$ & $297(76.35 \%)$ & 0.6432 & 0.4225 \\
\hline Chronic Obstructive Pulmonary Disease & $34(20.99 \%)$ & $47(20.7 \%)$ & $81(20.825)$ & 0.0046 & 0.946 \\
\hline Chronic Renal Insufficiency & $34(20.99 \%)$ & $47(20.7 \%)$ & $81(20.82 \%)$ & 0.0046 & 0.946 \\
\hline Urinary Tract Infection & $25(15.43 \%)$ & $22(9.69 \%)$ & $47(12.08 \%)$ & 2.9327 & 0.0868 \\
\hline Diabetes & $37(22.84 \%)$ & $52(23.01 \%)$ & $89(22.94 \%)$ & 0.0015 & 0.9688 \\
\hline
\end{tabular}

(\#=number, \%=percent of patients given protocol, $B M I=$ body mass index, $W=$ white $B=b l a c k, M=m a l e ~ F=f e m a l e, ~ P$ value=probability)

Table 1: New and Old method of care individual patient characteristics.

\begin{tabular}{|c|c|c|c|c|}
\hline $\begin{array}{l}\text { Patient Subgroups Characteristics Old } \\
\text { Protocol }\end{array}$ & Medicine & Ortho & Chi square & P value \\
\hline Number & 133 & 94 & & \\
\hline Mean Age & 81.75 (std dev 7.62) & 83.00(std dev 6.73) & & \\
\hline \multicolumn{5}{|l|}{ Number Age per Group } \\
\hline $65-75$ years & $29(21.80 \%)$ & $14(14.89 \%)$ & 1.7138 & 0.04245 \\
\hline $76-85$ years & $57(42.86 \%)$ & $44(46.81 \%)$ & & \\
\hline$>86$ years & $47(35.34 \%)$ & $36(38.30 \%)$ & & \\
\hline \multicolumn{5}{|l|}{ ASA } \\
\hline 1 & 0 & 0 & 18.002 & 0.0001 \\
\hline 2 & $5(3.76 \%)$ & $12(12.77 \%)$ & & \\
\hline 3 & $92(69.17 \%)$ & $75(79.79 \%)$ & & \\
\hline 4 & $36(27.07 \%)$ & $7(7.45 \%)$ & & \\
\hline 5 & 0 & 0 & & \\
\hline \multicolumn{5}{|l|}{ Fracture type } \\
\hline Intracapsular & $68(51.13 \%)$ & $45(47.87 \%)$ & 0.2335 & 0.629 \\
\hline
\end{tabular}


Citation: Brown CA, Mardam-Bey S, Boling J, Olson S, Owens T, et al. (2012) Review of Two Approaches for the Care of Elderly Hip Fracture Patient. J Trauma Treat 1:125. doi:10.4172/2167-1222.1000125

Page 4 of 7

\begin{tabular}{|c|c|c|c|c|}
\hline Extracapsular & $65(48.87 \%)$ & $49(52.13 \%)$ & & \\
\hline \multicolumn{5}{|l|}{ Pre-fracture Functional Status } \\
\hline Independent & $39(29.55 \%)$ & $40(44.44 \%)$ & 10.8669 & 0.0125 \\
\hline Cane & $26(19.70 \%)$ & $22(24.44 \%)$ & & \\
\hline Walker & $57(43.18 \%)$ & $27(30.0 \%)$ & & \\
\hline Wheelchair & $10(7.58 \%)$ & $1(1.11 \%)$ & & \\
\hline \multicolumn{5}{|l|}{ Preoperative Co-morbidities } \\
\hline Dementia & $45(33.83 \%)$ & $20(21.28 \%)$ & 4.2503 & 0.0392 \\
\hline Congestive Heart Failure & $46(34.59 \%)$ & $11(11.70 \%)$ & 15.3376 & $<0.001$ \\
\hline Coronary Heart Disease & $67(50.38 \%)$ & $26(27.66 \%)$ & 11.7516 & $<0.0006$ \\
\hline Peripheral Vascular Disease & $23(17.29 \%)$ & $4(4.26 \%)$ & 8.9336 & 0.0028 \\
\hline Cerebral Vascular Disease & $26(19.55 \%)$ & $14(14.89 \%)$ & 0.8222 & 0.3645 \\
\hline Hypertension & $106(79.70 \%)$ & $64(68.09 \%)$ & 3.9505 & 0.0469 \\
\hline Chronic Obstructive Pulmonary Disease & $37(27.82 \%)$ & $10(10.64 \%)$ & 9.9025 & 0.0017 \\
\hline Chronic Renal Insufficiency & $39(29.32 \%)$ & $8(8.51 \%)$ & 14.5308 & 0.0001 \\
\hline Urinary Tract Infection & $6(4.51 \%)$ & $16(17.02 \%)$ & 9.8479 & 0.0017 \\
\hline Diabetes & $40(30.30 \%)$ & $12(12.77 \%)$ & 9.5317 & 0.002 \\
\hline
\end{tabular}

Table 2: Subgroup Patient Characteristics prior to the New Protocol.

\begin{tabular}{|c|c|c|c|c|c|}
\hline Hospital Course & New \#(\% Approach) & Old \#(\% Approach) & Total \#(\%) & Chi Square & $P$ value \\
\hline Re-admission & $18(11.76 \%)$ & $34(15.32 \%)$ & $52(13.87 \%)$ & 0.956 & 0.3282 \\
\hline Rapid Response Code & $7(4.32 \%)$ & $12(5.29 \%)$ & $19(4.88 \%)$ & 0.1896 & 0.6632 \\
\hline Delirium & $61(37.65 \%)$ & $69(30.80 \%)$ & $130(33.68 \%)$ & 1.9753 & 0.1599 \\
\hline Myocardial Infarct & $14(8.64 \%)$ & $9(3.96 \%)$ & $23(5.91 \%)$ & 3.7175 & 0.0538 \\
\hline CHF exacerbation & $17(10.49 \%)$ & $21(9.25 \%)$ & $38(9.77 \%)$ & 0.1656 & 0.684 \\
\hline Pneumonia & $18(11.11 \%)$ & $16(7.05 \%)$ & $34(8.74 \%)$ & 1.9562 & 0.1619 \\
\hline COPD exacerbation & $10(6.17 \%)$ & $7(3.08 \%)$ & $17(4.37 \%)$ & 2.1586 & 0.1418 \\
\hline Pulmonary Embolus & $2(1.23 \%)$ & $6(2.64 \%)$ & $8(2.06)$ & 0.9312 & 0.3345 \\
\hline DVT & $6(3.70 \%)$ & $7(3.08 \%)$ & $13(3.34 \%)$ & 0.1125 & 0.7373 \\
\hline Decubitus Ulcer & $6(3.70 \%)$ & $4(1.76 \%)$ & $10(2.57 \%)$ & 1.4229 & 0.2329 \\
\hline Surgical wound & $8(4.94 \%)$ & $7(3.08 \%)$ & $15(3.86 \%)$ & 0.877 & 0.349 \\
\hline Transfusion & $84(51.85 \%)$ & $83(36.56 \%)$ & $167(42.93 \%)$ & 9.0182 & 0.0027 \\
\hline Urinary Tract Infection & $17(10.49 \%)$ & $36(15.93 \%)$ & $53(13.66 \%)$ & 2.3637 & 0.1242 \\
\hline Renal Insufficiency & $38(23.46 \%)$ & $35(15.42 \%)$ & $73(18.77 \%)$ & 4.0069 & 0.0453 \\
\hline Death & $7(4.32 \%)$ & $4(1.76 \%)$ & $11(2.83 \%)$ & 2.2527 & 0.1334 \\
\hline
\end{tabular}

(\#=number, \%=percent of patients given protocol, $\mathrm{CHF}=$ congestive heart failure, COPD=chronic obstructive pulmonary disease, $\mathrm{DVT}=$ deep venous thrombosis)

Table 3: New vs. Old method of care hospital course, perioperative complications, and mortality.

\begin{tabular}{|c|c|c|c|c|}
\hline Hospital Course & Medicine & Orthopedics & Chi Square & P value \\
\hline Re-admission & $24(18.60 \%)$ & $10(10.75 \%)$ & 2.5689 & 0.1090 \\
\hline Rapid Response Code & $10(7.52 \%)$ & $2(2.13 \%)$ & 3.1970 & 0.0738 \\
\hline Delirium & $47(35.88 \%)$ & $22(23.66 \%)$ & 3.8116 & 0.0509 \\
\hline Myocardial Infarct & $8(6.02 \%)$ & $1(1.06 \%)$ & 3.5459 & 0.0597 \\
\hline CHF exacerbation & $17(12.78 \%)$ & $4(4.26 \%)$ & 4.7695 & 0.0290 \\
\hline Pneumonia & $14(10.53 \%)$ & $2(2.13 \%)$ & 5.9296 & 0.0149 \\
\hline COPD exacerbation & $7(5.26 \%)$ & 0 & 5.1048 & 0.0239 \\
\hline Pulmonary Embolus & $5(3.76 \%)$ & $1(1.06 \%)$ & 1.5551 & 0.2124 \\
\hline DVT & $3(2.26 \%)$ & $4(4.26 \%)$ & 0.7369 & 0.3907 \\
\hline Decubitus Ulcer & $4(3.07 \%)$ & 0 & 2.8778 & 0.0898 \\
\hline Surgical wound & $3(2.26 \%)$ & $4(4.26 \%)$ & 0.7369 & 0.3907 \\
\hline Transfusion & $46(34.59 \%)$ & $37(39.36 \%)$ & 0.5414 & 0.4618 \\
\hline Urinary Tract Infection & $26(19.70 \%)$ & $10(10.64 \%)$ & 3.3642 & 0.0666 \\
\hline Renal Insufficiency & $26(19.70 \%)$ & $9(9.57 \%)$ & 4.2016 & 0.0404 \\
\hline Death & $3(2.26 \%)$ & $1(1.06 \%)$ & 0.4519 & 0.5014 \\
\hline
\end{tabular}

(\#=number, \%=percent of patients given protocol, CHF=congestive heart failure, COPD=chronic obstructive pulmonary disease, DVT=deep venous thrombosis)

Table 4: Peri-operative complications and mortality in Medicine and Orthopedic patients prior to the new protocol. 
subgroups initially admitted under the OP we found that the TTS and LOS for those admitted to medicine was greater than those admitted to orthopedics (Table 5b). Orthopedic TTS was 20.72 (std dev 21.27) hrs and LOS was 5.83 (std dev 2.65) and Medicine TTS was 31.13 (std dev 33.12) hrs and LOS 7.55 (std dev 5.36) days.

Table 6 illustrates that the NP also had a significant increased frequency of patients being recognized as having Vitamin D deficiency and being supplemented when compared to the OP $(42.12 \%$ vs. $20.70 \%)$.

\section{Discussion}

The impact of waiting time for surgery on outcome has been studied with a main emphasis on mortality, length of hospital stay, and postoperative complications [15-21].

The barriers to delays in surgery are many and at times are difficult to get around and include lack of theatre capacity, need for timely medical optimization, poor ward management, co-existing medical conditions, and lack of equipment. Guryel et al. [22] who found that medical reasons and waiting times for such things as preoperative echocardiography delayed patients more frequently than a lack of theatre availability. Timely preoperative evaluation, medical optimization and surgery should be associated with decreased perioperative complications and improved outcomes. Therefore, the time-consuming admission paper work and interplay between services needs to be minimized and optimized to prevent the possible negative effect of a prolonged waiting time to surgery.

Studies with internal medicine have shown efficiency of hospitalists by reduction of LOS and cost $[18,19,23]$. Medical literature describes improved outcomes and efficiency when an intensivist or geriatrician participates in treatment of elderly patients [20,21,24]. Expertise gained by a geriatrician and medical physician in dealing with medical co-morbidities in these surgical patients may help to explain improved outcomes. Despite these encouraging reports by medial physicians it is still unclear how to best form an efficient care plan to help reduce the morbidity and mortality that translates to decreased hospital stay and costs.

We had anticipated that by direct admission to medical service we would help to facilitate preoperative medical evaluation, promote medical optimization in preparation for surgery and decrease TTS Although the results of the NP did show a decrease in mean TTS surgery of 2.57 hours this was not statistically significant. Both the NP and OP TTS are comparable to prior reports ranging from 1.3-3.6 days [25].

This is explained by our subgroup analysis which demonstrates that our "sicker" patients were admitted to Medicine in both protocols. Patients on the medicine service under the OP had an increased TTS to begin with when compared to those that went to orthopedics. While many patients with multiple co-morbidities were admitted to orthopedics under the OP, the change in care plans did not reflect a decreased TTS. Therefore, no differences were likely found in TTS because the patients in which a change in care occurred under the NP were on average healthier.

We had also hoped that with the increase in prevalence of chronic disease and medical complexity that some of these patients LOS would be decreased with the NP. However, the LOS was not significantly different between either the earlier or new approach. The NP mean LOS 7.15 (std dev 4.26) days and the OP mean LOS 6.83 (std dev 4.52) days. Similar to TTS, this is likely explained by our results in that a greater number of patients initially admitted to medicine under the OP were less healthy and had increased frequency of certain peri-operative complications. This association is reflected in the increased LOS of those patients initially cared for by the medicine service.

Some reports indicate an association between a delay of surgery and the length of hospitalization. Siegmeth et al. [26] noted that a delay of 7.85 hours to the time of surgery was associated with an increased hospital stay of one day. Another study reported a similar finding that with every 6.75-hour delay to surgery the total length of the hospital

\begin{tabular}{|c|c|c|c|c|c|c|}
\hline Protocol & Mean TTS (hrs) & $95 \% \mathrm{Cl}$ & std dev & Mean LOS(days) & $95 \% \mathrm{Cl}$ & std dev \\
\hline New & 24.45 & $20.14-28.76$ & 27.8 & 7.15 & $6.48-7.82$ & 4.26 \\
\hline Old & 27.02 & $22.91-31.11$ & 29.41 & 6.83 & $6.24-7.42$ & 4.52 \\
\hline Total & 25.87 & & 29.69 & 6.96 & & 4.41 \\
\hline T value (prob) & $(-) 0.85(0.3952)$ & & & $0.70(0.4846)$ & & \\
\hline
\end{tabular}

(TTS=time to surgery, LOS=length of stay, hrs=hours, $\mathrm{Cl}=$ confidence interval, std dev=standard deviation, $\mathrm{T}$ value (prob)=student $\mathrm{t}$ test and probability)

Table 5a: New vs. Old method of care TTS and LOS.

\begin{tabular}{|c|c|c|c|c|c|c|}
\hline Protocol & Mean TTS (hrs) & $95 \% \mathrm{Cl}$ & std dev & Mean LOS(days) & $95 \% \mathrm{Cl}$ & std dev \\
\hline Medicine & 31.13 & $25.17-37.09$ & 33.12 & 7.55 & $6.62-8.47$ & 5.36 \\
\hline Orthopedics & 20.72 & $18.95-25.48$ & 21.27 & 5.83 & $5.28-6.38$ & 2.68 \\
\hline \multicolumn{7}{|l|}{ Total } \\
\hline T value (prob) & $16.17(<0.0001)$ & & & $10.34(<0.0001$ & & \\
\hline
\end{tabular}

(TTS=time to surgery, LOS=length of stay, hrs=hours, $\mathrm{Cl}=$ confidence interval, std dev=standard deviation, $\mathrm{T}$ value (prob)=student $\mathrm{t}$ test and probability

Table 5b: Subgroup TTS and LOS for Medicine and Orthopedic patients prior to the New Protocol.

\begin{tabular}{|c|c|c|c|c|}
\hline Supplementation & New \# (\% Approach) & Old \# (\% Approach) & Chi square & P value \\
\hline $\begin{array}{r}\text { Newly Diagnosed Vitamin D } \\
\text { Deficiency }\end{array}$ & $65(42.12 \%)$ & $47(20.70 \%)$ & 17.3872 & $<0.0001$ \\
\hline $\begin{array}{r}\text { Already on Vitamin D or Never } \\
\text { Diagnosed }\end{array}$ & $97(59.87 \%)$ & $180(79.30 \%)$ & & \\
\hline Total & 162 & 227 & & \\
\hline
\end{tabular}

(\#=number, \%=percent of patients given approach)

Table 6: New vs. Old method of care management of Vitamin D Deficiency. 
stay was increased by one day [27]. Another potential explanation is that TTS and LOS may ultimately be governed by patient pre-fracture characteristics such as ASA, age, co-morbidities, fracture type, or prior level of ambulatory status and not admission service. In the end, both the NP and OP LOS results are encouraging as hospital stay for both approaches fall within the lower range of prior reported results ranging from 4.6-47 days [25].

Elderly patients who experience a hip fracture have been shown to have an increased in-hospital mortality rates ranging from 1.14-20\% [25]. In our study, both the NP and OP rates fell well within this range and did significantly differ from one another (Table 3). Our study is not powered to detect a difference in mortality, as the total number of inpatient deaths was only 11 (2.83\% mortality rate). We also only recorded deaths that occurred during concurrent admission such that mortality rates at 30 days or 1 year may be a better predictor of the impact a hip fracture has on these patients. Reports support this and indicate that 30 day and 1 year morality are likely increased when compared to in-hospital mortality [25].

We found that there were no significant differences in perioperative complications between the NP and the OP in regard to 30 day readmission, RRT, postoperative delirium, MI, CHF exacerbation, pneumonia, COPD exacerbation, PE, DVT, decubitus ulcers, surgical wound complications, UTI. We did find a difference in the number of patients that were transfused between the NP and OP. Approximately $15 \%$ more patients were transfused under the NP. Although, indications for transfusion at our hospital have standard guidelines and criteria, the clinical decision to transfuse ultimately falls on the admitting team. From this analysis, it would appear that when all patients were admitted to medicine under the NP that there was an increase in the number of transfusions. It is unclear as to why this occurred as both the OP and NP had similar distribution of chronological aged patients, comorbidities, and intracapsular vs extracapsular type of fractures.

We initiated this new protocol as a patient safety initiative due to the perception that patient care was delayed in the choice of admission service and that complications were occurring on the orthopedic service which could be more safely handled by the medicine service. Actually, our subgroup analysis showed the opposite. In fact, patients initially admitted to orthopedics under the OP had a decreased frequency of certain peri-operative complications such as CHF exacerbation, COPD exacerbation, pneumonia, and renal insufficiency. However, despite the sicker patients being initially admitted to medicine there was not a significant difference in MI, PE, DVT, decubitus ulcers, wound complications, UTI, delirium, RRT, re-admission, or death. That is, the OP orthopedic patients were healthier, but not without disease or perioperative complications. Until this review one could have argued that at our institution improvements in care, TTS or LOS may have resulted from a protocol in which each service was more familiar with their roles from admission and with the management of complex comorbidities. It is reassuring that we were providing and continue to provide the safest care possible to our patients under both protocols.

In the United States, although approximately $90 \%$ of patients with hip fractures have osteoporosis, only $19 \%$ of them receive post fracture evaluation and therapy [28]. We used the supplementation of Vitamin $\mathrm{D}$ and calcium on an inpatient basis as an index of implementing a preventive strategy. It is general practice at out institution that after diagnosis with Vitamin D deficiency a bone density scan is generally done in an outpatient setting after discharge for additional osteoporosis evaluation if needed. We found that the NP demonstrated a significant number of patients being recognized with Vitamin D deficiency and being discharged on supplementation when compared to the OP (42.12\% vs. $20.70 \%$ ). This reaffirms the importance of incorporating Vitamin D recommendations in to fracture care pathways [29].

Implementing preventative strategies will likely play a crucial role in decreasing future numbers of fractures and cost containment. Strategies that allow earlier recognition of osteoporosis and implementation of both life style changes and medical management are thought to have been beneficial to this endeavor. A simple computerassisted reminder in the Brigham fracture intervention team initiative was found to increase the effectiveness of evaluating osteoporosis by $68 \%$ [29]. According to one model the cost per hip fracture avoided is $\$ 48,600$ if a 62 -year-old woman with osteoporosis receives treatment with a drug that is administered for 5 years at $\$ 830$ /year and produces a $50 \%$ reduction in fracture rate. The cost per life-year saved is $\$ 30,600$, and the cost per quality-adjusted life-year is $\$ 14,900$ [1]. Deciphering which patients will benefit most from preventive strategies will be key to future reduction of fractures. Future research and funding into developing more standardized cost-effective preventive and medical care pathways for these patients will be needed as the global projected hip fracture incidence in men and women may increase by $200-300 \%$ by the year 2050 [30].

We acknowledge several limitations of this retrospective study. It is likely that no differences in perioperative complications, TTS, or LOS were found when we admitted all patients to medicine because the only difference in care occurred for those patients who fell in the mid spectrum to healthy. The patients with more co-morbidities and increased frequency of peri-operative complications were already being admitted too and cared for by a medicine team. Our hospital is not a high volume hip unit and thereby, our study is underpowered. Data also were collected through a chart review of electronic medical records such that the data were limited to the quality of chart documentation. This analysis is limited to the experience of our academic institution and therefore may not be generalizable beyond our hospital.

In conclusion, the ideal model of care for hip fracture patients is unknown. We altered our model of care to a protocol where all patients over 65 with a hip fracture were admitted to the medicine service to try and improve patient safety. While our change in protocol did not alter the rate of perioperative complications, nor did it provide quicker care or shorter hospital stays, it did provide more comprehensive osteoporosis care to our patients. This report has provided information to our institution to further look at more evidence based algorithms that may lead to improved admission and care of the hip fracture patient.

\section{References}

1. Johnell O (1997) The socioeconomic burden of fractures: today and in the 21st century. Am J Med 103: 20S-25S.

2. Brown CA, Starr AZ, Nunley JA (2012) Analysis of past secular trends of hip fractures and predicted number in the future 2010-2050. J Orthop Trauma 26 : 117-122.

3. http://www2.aaos.org/aaos/archives/acadnews/2000news/c9-17.htm

4. Roy A, Heckman MG, Roy V (2006) Associations between the hospitalist mode of care and quality-of-care-related outcomes in patients undergoing hip fracture surgery. Mayo Clin Proc 81: 28-31.

5. Phy MP, Vanness DJ, Melton LJ 3rd, Long KH, Schleck CD, et al. (2005) Effects of a hospitalist model on elderly patients with hip fracture. Arch Intern Med 165: 796-801.

6. Elliot JR, Wilkinson TJ, Hanger HC, Gilchrist NL, Sainsbury R, (1996) The added effectiveness of early geriatrician involvement on acute orthopaedic wards to orthogeriatric rehabilitation. N Z Med J 109:72-73. 
Citation: Brown CA, Mardam-Bey S, Boling J, Olson S, Owens T, et al. (2012) Review of Two Approaches for the Care of Elderly Hip Fracture Patient. J Trauma Treat 1:125. doi:10.4172/2167-1222.1000125

Page 7 of 7

7. Roberts HC, Pickering RM, Onslow E, Clancy M, Powell J, (2004) The effectiveness of implementing a care pathway for femoral neck fracture in older people: a prospective controlled before and after study. Age Ageing 33: 178184.

8. Fisher AA, Davis MW, Rubenach SE, Sivakumaran S, Smith PN, (2006) Outcomes for older patients with hip fractures: the impact of orthopedic and geriatric medicine cocare. J Orthop Trauma 20: 172-180.

9. Huddleston JM, Long KH, Naessens JM, Vanness D, Larson D, (2004) Medical and surgical comanagement after elective hip and knee arthroplasty: a randomized, controlled trial. Ann Intern Med 141: 28-38.

10. Khasraghi FA, Christmas C, Lee EJ, Mears SC, Wenz JF Sr (2005) Effectiveness of a multidisciplinary team approach to hip fracture management. J Surg Orthop Adv 14: 27-31.

11. Vidán M, Serra JA, Moreno C, Riquelme G, Ortiz J (2005) Efficacy of a comprehensive geriatric intervention in older patients hospitalized for hip fracture: a randomized, controlled trial. J Am Geriatr Soc 53: 1476-1482.

12. Naglie G, Tansey C, Kirkland JL, Ogilvie-Harris DJ, Detsky AS, (2002) Interdisciplinary inpatient care for elderly people with hip fracture: a randomized controlled trial. CMAJ 167: 25-32.

13. Tosi LL, Kyle RF (2005) Fragility fractures: the fall and decline of bone health. Commentary on "Interventions to improve osteoporosis treatment following hip fracture" by Gardner et Al. J Bone Joint Surg Am 87: 1-2.

14. Owens WD, Felts JA, Spitznagel EL Jr (1978) ASA physical status classifications: a study of consistency of ratings. Anesthesiology 49: 239-243.

15. Zuckerman JD, Skovron ML, Koval KJ, Aharonoff G, Frankel VH (1995) Postoperative complications and mortality associated with operative delay in older patients who have a fracture of the hip. J Bone Joint Surg Am 77: 15511556.

16. Parker MJ, Pryor GA (1992) The timing of surgery for proximal femoral fractures. J Bone Joint Surg Br 74: 203-205.

17. Orosz GM, Magaziner J, Hannan EL, Morrison RS, Koval K, (2004) Association of timing of surgery for hip fracture and patient outcomes. JAMA 291: 17381743.

18. Kaboli PJ, Barnett MJ, Rosenthal GE (2004) Associations with reduced length of stay and costs on an academic hospitalist service. Am J Manag Care 10: 561-568.
19. Hackner D, Tu G, Braunstein GD, Ault M, Weingarten S, (2001) The value of a hospitalist service: efficient care for the aging population? Chest 119: 580-589.

20. Manthous CA, Amoateng-Adjepong Y, al-Kharrat T, Jacob B, Alnuaimat HM (1997) Effects of a medical intensivist on patient care in a community teaching hospital. Mayo Clin Proc 72: 391-399.

21. Whitaker JJ, Currie CT (1989) Non-orthopaedic problems in the elderly on an acute orthopaedic unit: the case for geriatrician input. Health Bull (Edinb) 47 72-77.

22. Guryel E, Redfern DJ, Ricketts DM (2004) Balancing priorities in the management of hip fractures: guidelines versus resources. Ann R Coll Surg Engl 86: 171-173.

23. Davis KM, Koch KE, Harvey JK, Wilson R, Englert J, et al. (2000) Effects of hospitalists on cost, outcomes, and patient satisfaction in a rural health system. Am J Med 108: 621-626.

24. Murphy PJ, Rai GS, Lowy M, Bielawska C (1987) The beneficial effects of join orthopaedic-geriatric rehabilitation. Age Ageing 16: 273-178.

25. Kammerlander C, Roth T, Friedman SM, Suhm N, Luger TJ, et al. (2010) Orthogeriatric service--a literature review comparing different models. Osteoporos Int 21: S637-646.

26. Siegmeth AW, Gurusamy K, Parker MJ (2005) Delay to surgery prolongs hospital stay in patients with fractures of the proximal femur. J Bone Joint Surg $\mathrm{Br}$ 87: 1123-1126.

27. Al-Ani AN, Samuelsson B, Tidermark J, Norling A, Ekström W, et al. Early operation on patients with a hip fracture improved the ability to return to independent living. A prospective study of 850 patients. J Bone Joint Surg Am 90: 1436-1442.

28. (2008) The State of Healthcare Quality, "Industry Trends and Analysis. National Commitee for Quality Assurance Health Plan Employer Data and Information Set".

29. Glowacki J, Harris MB, Simon J, Wright J, Kolatkar NS, (2010) Brigham fracture intervention team initiatives for hospital patients with hip fractures: a paradigm shift. Int J Endocrinol 2010: 590751

30. Gullberg B, Johnell O, Kanis JA (1997) World-wide projections for hip fracture. Osteoporos Int 7: 407-413. 